\title{
Randomized controlled trials in surgery: Comic opera no more?
}

\author{
Neal F. Kassell, MD, and Aaron S. Dumont, MD
}

See related articles on pages 229, 233, 241, 245, 247, and 249.
From the Department of Neurological Surgery, University of Virginia School of Medicine, Charlottesville, Va.

Received for publication Jan 4, 2006; accepted for publication Mar 3, 2006.

Address for reprints: Neal F. Kassell, MD, Box 800212, Department of Neurological Surgery, UVA Health Sciences Center, Charlottesville, VA 22908 (E-mail: nfk8g@ virginia.edu).

J Thorac Cardiovasc Surg 2006;132:243-4

$0022-5223 / \$ 32.00$

Copyright $(C) 2006$ by The American Association for Thoracic Surgery

doi:10.1016/j.jtcvs.2006.03.041
$\mathrm{T}$

The ultimate goal of clinical research is to provide the best information to optimally guide the management of a given patient with a particular problem confronting us. Specifically, surgeons might wish to compare medications, surgical techniques, or combinations thereof for a given condition. Randomized controlled trials (RCTs) help to circumvent several major obstacles hindering investigations of this nature. Through randomization, selection bias is theoretically removed, and confounding factors are distributed evenly among groups to mitigate their potential influences. Hence RCTs have emerged as the most efficient and rigorous way to generate robust scientific evidence of safety and efficacy necessary to prove whether a therapeutic intervention works.

However, such trials are expensive, time consuming, difficult to perform, and potentially dangerous. They are obviously not the only way to determine whether a given therapeutic intervention is efficacious.

Surgery has been progressing for thousands of years, with effective procedures being adopted and ineffective procedures discarded without the benefit of RCTs, which are a relative newcomer.

From a pragmatic perspective, many surgical disorders and procedures are not amenable to study by RCTs because of small numbers, which make the length of time required to accrue an adequate number of cases too long. Indeed, critical analyses of negative results of RCTs published in the surgical literature demonstrate that many reports with negative results lack sufficient statistical power to definitively support or refute the null hypothesis at hand. ${ }^{1}$ Furthermore, these difficulties are compounded by the acceleration and evolution of technology.

There is a hierarchy in the development of information to decide whether an intervention is beneficial. Anecdotal evidence from a small number of patients is provided by case reports. Suggestive evidence is provided by case series, usually retrospective analysis from a single institution. Supportive evidence is provided by observational studies-open trials-epidemiologic surveys of a large number of cases from many institutions where data are collected prospectively in a standardized format. Lastly, there is the sage advice of a wise and experienced surgeon. This should not be neglected in our compulsion to worship at the altar of more rigorous scientific studies.

Before embarking on a randomized clinical trial, there must be adequate supportive evidence not only concerning the safety and effectiveness of the procedure but also of the group that is serving as the baseline. This supportive evidence is provided by the epidemiologic survey open trial or observational study. In many instances a rigorously conducted epidemiologic survey will obviate the need for a randomized trial. And, as noted above, in many instances of surgical procedures, randomized clinical trials are simply not feasible.

The following are caveats applicable to the design and conduct of RCTs:

- Sample size. No trial ever failed because of too many cases.

- Exploratory analysis. Not infrequently, the most valuable data about the procedure or about the disease being studied will be found in subgroups during exploratory analyses that were not anticipated at the time of study design. This improves data information about the patient population and their disease, as well as the procedure. 
- Organization. Beware of trials of surgical procedures organized by nonsurgeons.

- Outcome measures. Despite pressure from regulatory authorities to choose a single end point, in reality multiple end points are important. This includes compound end points involving a combination of end points. End points need to be evaluated by individuals who are blind to the procedure and independent to the management of the patients. Ideally, they should be individuals who have no vested interest in whether the procedure being studied works.

It is also important to heed the key messages conveyed by Tiruvoipati and colleagues ${ }^{2}$ in the current article. In neurosurgery, particularly in certain complex cranial base cases, we emphasize that it is important to plan the closure before the opening. Similarly, planning of the reporting of an RCT during all phases of the trial is important. Attention to all of these aforementioned details and improvement in the quality of reporting as we move forward might help to make the "comic opera" stigma ${ }^{3}$ associated with research in surgery a thing of the past.

\section{References}

1. Dimick JB, Diener-West M, Lipsett PA. Negative results of randomized clinical trials published in the surgical literature. Equivalency or error? Arch Surg. 2001;136:796-800.

2. Tiruvoipati R, Balasubramanian SP, Atturu G, Peek GJ, Elbourne D. Improving the quality of reporting randomized controlled trials in cardiothoracic surgery: the way forward. $J$ Thorac Cardiovasc Surg. 2006;132:233-40.

3. Horton R. Surgical research or comic opera: questions but few answers. Lancet. 1996;347:984-5. 